\title{
Acoplamiento entre Fotons-Plasmons a través de iones de tierras raras y superficies de plasmons localizados
}

Photon-Plasmons coupling via rare earth ions and lozalized surfaces plasmons

Recibido: marzo 11 de 2017 | Revisado: abril 10 de 2017 | Aceptado: mayo 15 de 2017

V.A.G. RIVERA

Resumen

La superficie plasmons permite la localización de la luz a escala nanométrica lo cual nos proporciona una manera eficaz de controlar la excitación y las propiedades de los sistemas cuánticos. La confinación de los campos electromagnéticos en regiones pequeñas permite diversas aplicaciones tecnológicas, tales como nanofotónica, biosensoramiento, biotecnología, imágenes para medicina y otros excitantes campos tecnológicos. En ese sentido, la fabricación de nano-estructuras que puedan sostener la superficie de los plasmons puede excitar emisores cuánticos y hasta modificar las emisiones de estos a través de cambios en la densidad de población de un determinado nivel de energía del emisor cuántico. Un ejemplo de estos emisores cuánticos son los iones de tierras raras, los cuales a través de un acoplamiento fotón-plasmón permite nuevos desarrollos tecnológicos.

\section{Palabras Clave:}

\begin{abstract}
Surface plasmons enable the localization of light on nanoscale and provide an effective way of controlling the excitation and emission properties of quantum systems. The confinement of electromagnetic fields in regions as small as possible is important for diverse applications, such as nanophotonics, biosensing, biotechnology, medical imaging and others. In this sense, metallic nanostructures sustaining surface plasmons can change the excitation and emission properties of locally excited quantum systems, such as rare-earth ions resulting in an interaction plasmon-photon that enable new technologic applications.
\end{abstract}

Key words: localized surface plasmon, rare-earth ions,

\section{plasmonics, photonics}

\footnotetext{
1 Universidad Nacional Mayor de San Marcos, Lima, Perú vgarciar1@unmsm.edu.pe
}

https://doi.org/10.24265/ campus.2017.v22n23.10

| Campus | Lima, perú | V. XXII | N. 23 | PP. I29- I32 | ENERO-JUNIO | 20I7 | ISSN I8I 2-6049 
Plasmónica (Editorial Note, Surface plasmon resurrection, Nature Photonics Vol. 6 (11) 707) es una interesante y prometedora área de investigación de frontera tanto en la nanociencia como en la nanotecnología, que puede llevar a la electrónica y fotónica (Quimby, 2006) a escala nanométrica proporcionándonos una verdadera solución a la integración y transferencia de datos a esa nanoescala. La intersección de esas dos grandes áreas de investigación es conocida como nanofotónica (Rigneault, Loutioz,Delalande \& Levenson, 2010) es una de las áreas del conocimiento de frontera y del desarrollo de tecnologías de punta, ya que estratégicamente aborda la interacción de la luz con la materia por debajo del límite de difracción (Gaponenko, 2010; Rivera, Ferri\& Marega, 2012) y puede potencialmente revolucionar los conceptos de las telecomunicaciones ópticas que conocemos hoy en día. Esto es debido a la capacidad que poseen los nanodispositivos plasmónicos de confinar la luz en volúmenes nanométricos por debajo del límite de difracción, estos campos electromagnéticos confinados pueden interactuar con emisores cuánticos.

Uno de los emisores cuánticos más prometedores son los iones de tierras raras (REIs, del inglés rare-earth ions) o lantánidos. Son un grupo de elementos que forman parte del periodo 6 de la tabla periódica de los elementos. Los REIs son elementos promisorios debido a sus características magnéticas, luminiscentes y electroquímicas. Se emplean en la fabricación de nuevos dispositivos debido a su mayor eficiencia, alta velocidad, mayor durabilidad y su potencial para procesos de miniaturización. En esta ruta de miniaturización, la nanofotónica no es solo el diseño y fabricación de dispositivos fotónicos a nanoescala, es también nuevas formas de manipular el flujo de la luz por medio de esas nanoestructuras, procesos de transferencia de energía, entre otros. Los electrones que son responsables de las propiedades de los REIs son electrones $4 \mathrm{f}$. Estos orbitales $4 \mathrm{f}$ están protegidos de la influencia de fuerzas externas por las capas externas $5 s^{2}$ y $5 \mathrm{p}^{6}$. Es por ello que las diversas configuraciones $4 \mathrm{f}^{\mathrm{n}}$ solo son afectadas ligeramente por el medio que rodea a los iones y permanecen prácticamente inalterables para determinado ion en todos sus compuestos.

El resultado de la unión de los dispositivos plasmónicos y los REIs son las fascinantes propiedades ópticas que nos conllevan a manipular los estados cuánticos de los REIs y controlar su emisión e intensidad de radiación óptica a nanoescala, con innumerables aplicaciones prácticas en telecomunicaciones, energía limpia, biofotónica y pruebas médicas.

De hecho, la superficie de plasmón (SP), son las oscilaciones de electrones libres que se encuentran sobre la superficie metálica. Estos pueden crear ondas eléctricas o magnéticas evanescentes propagantes e incluso penetrar la superficie metálica (generalmente del orden de unas decenas de nanómetros), llegando a varios micrómetros del sustrato (generalmente un material dieléctrico). Esta SP hoy en día ha permitido desarrollar nuevas tecnologías más allá de las escalas de las longitudes de onda, ya que dentro de sus bondades tecnológicas pueden generar transferencia de energía, amplificación de intensidad eléctrica en varias decenas de amplificación del campo 
eléctrico local (Rivera, Ferri \& Marega, 2017).

Dentro de esta distancia de penetración, podemos tener que un REI representado por un sistema cuántico de dos niveles de energía, es decir, solo es permitida una transición electrónica, la cual puede generar un campo electromagnético el cual puede interactuar fuertemente con la SP, al cual llamaremos I-SP, así como también puede interactuar entre la interface metal dieléctrico. La frecuencia de estos I-SP corresponde a la frecuencia de emisión del REI, por lo tanto, sus emisiones tienen la misma forma espectral y están altamente polarizadas como resultado de la polarización inducida y localizada del SP (LSP).
Estas interacciones colectivas de fotón-plasmón (Hieu \& Ha, 2015) están siendo estudiadas desde el punto de vista teórico (Goldman, 1966) como experimental (V.A.G. Rivera, 2012) tendrán un impacto positivo o negativo para un sistema cuántico electrónico sobre el REI y dependerá de la eficiencia del acoplamiento de estos sistemas. De esta manera, tendremos la capacidad de diseñar una manera de contrarrestar las pérdidas en el metal (efectos de dispersión de energía), modular proceso de emisión de conversión hacia abajo o de conversión hacia arriba del REIs (V.A.G. Rivera, 2012, Richard S. Quimby, 2006), controlar la polarización/fase de la emisión de los REIs permitiendo una diversidad de aplicaciones tecnológicas.

\section{Referencias}

Gaponenko, S.V. (2010). Introduction to Nanophotonics. USA: Cambridge University Press.

Rigneault, H., Lourtioz, J.M., Delalande, C. \& Levenson, A. (2010). Nanophotonics. USA: Wiley.

Goldman, M.V. (1966). Parametric plasmon-photon interaction: Part I. Threshold for amplification of plasmons. Annals of Physics, 38(1), 95-116.

Quimby, R. (2006). Photonics and Lasers: An Introduction. USA: Wiley.

Rivera, V.A.G., Ferri, F.A. \& Marega, E. (2012). Localized Surface Plasmon Resonances: Noble Metal Nanoparticle Interaction with Rare-Earth Ions. En K. Young (Ed.), Plasmonics - Principles and Applications. Croatia: InTech. doi:10.5772/50753.
Rivera, V.A.G., Ferri F.A. \& Marega E. (2017). Tellurite Glasses for Plasmonics. En V. Rivera, D. Manzani (Eds.), Technological Advances in Tellurite Glasses (Vol. 254). Alemania: Springer.

V.A.G. Rivera, Silva OB, Ledemi Y, Messeddeq Y, Marega E Jr 2015, Collective plasmon modes in gain media. Quantum Emitters and Plasmonic Nanostructures Series 25, pp. 28.

Hieu, V. \& Ha, B. (2015). Quantum field theory of interacting plasmon-photon system, Adv. Nat. Sci.: Nanosci. Nanotechnol, 6(2). Recuperado de http://iopscience. iop.org/article/10.1088/2043-62 62/6/2/025010 
